\title{
Video Article \\ Chemical Isolation, Quantification, and Separation of Skin Lipids from Reptiles
}

\author{
Paige E. Baedke ${ }^{1}$, Holly R. Rucker ${ }^{1}$, Robert T. Mason ${ }^{2}$, M. Rockwell Parker ${ }^{1}$ \\ ${ }^{1}$ Department of Biology, James Madison University \\ ${ }^{2}$ Department of Integrative Biology, Oregon State University
}

Correspondence to: M. Rockwell Parker at mrockwellparker@gmail.com

URL: https://www.jove.com/video/59018

DOI: doi: $10.3791 / 59018$

Keywords: Environmental Sciences, Issue 144, Pheromone, analytical chemistry, GC-MS, chromatography, reptile, snake, extraction, chemical ecology, alumina

Date Published: 2/7/2019

Citation: Baedke, P.E., Rucker, H.R., Mason, R.T., Parker, M.R. Chemical Isolation, Quantification, and Separation of Skin Lipids from Reptiles. J. Vis. Exp. (144), e59018, doi:10.3791/59018 (2019).

\section{Abstract}

Reptiles signal to conspecifics using lipids in their skin, primarily to enable mate tracking and assessment. The isolation of these lipids has utility in research focused on evolutionary patterns and mechanisms of chemical communication, in addition to understanding the waterproofing role of lipids in the evolution of terrestrial life. In an applied approach, such skin-based cues have potential use for wildlife managers dealing with invasive species. The main steps for quantifying reptile skin lipids in the protocol presented here include extraction, total lipid determination, and fractionation via column chromatography, the latter process resulting in purified eluates of compounds which can then either be analyzed to assign compound identifications (e.g., gas chromatography-mass spectrometry [GC-MS]) and/or used directly in more refined bioassays. Skin lipids can be extracted from living skin, shed skin, or dead whole animals, using nonpolar organic solvents (e.g., hexane, benzene, toluene). Extraction solubilizes the lipids and, then, the solvent can be evaporated to yield a measurable lipid-only extract. Fractionation involves the separation of the total lipid extract into specific eluates via traditional column chromatography. The total lipid extract is first bound to a substratebased column (e.g., alumina) and, then, individual eluates ("fractions") of solvent at specific volumes are passed sequentially through the column to elute sets of compounds from the lipid mixture based on common polarity. The fractions progress in polarity at a standardized sequence by increasing the relative amount of polar solvent (e.g., diethyl ether) in nonpolar solvent. In this manuscript, we describe several methods for extracting skin lipids of reptiles and, then, provide a standard protocol for isolating different sets of compounds based on polarity, using traditional column chromatography. Whole lipid extracts or specific fractions can, then, be used in bioassays to determine any biological activity elicited by the compounds therein.

\section{Video Link}

The video component of this article can be found at https://www.jove.com/video/59018/

\section{Introduction}

Reptiles produce lipids in the epidermis, either directly from skin cells or from discrete glands that are used in social communication, such as mate assessment and tracking, territoriality, and intra- and interspecific recognition ${ }^{1,2,3,4}$. The isolation of these skin lipids has utility in research focused on evolutionary patterns and mechanisms of chemical communication, in addition to understanding the waterproofing role of lipids in the evolution of terrestrial life $e^{2,3,4}$. Further, many reptiles, especially squamates (lizards, snakes), are invasive species of concern in sensitive ecosystems, and the development of pheromone-based lures to improve trapping and removal is ongoing ${ }^{5,6}$. The impermeability of reptile skin facilitates the extraction of the lipids present to obtain relatively pure extractions of a potentially robust source of chemical signals. The principle steps for quantifying reptile skin lipids in the described protocol include extraction, total lipid determination, and fractionation via column chromatography ${ }^{1,6,7}$. The methods have been used routinely as they yield bioactive isolates that explain much about mate choice and selection, especially in snakes ${ }^{2}$.

Skin lipids can be extracted from either living skin, shed skin, or dead whole reptiles, using nonpolar or polar organic solvents ${ }^{1,7,8,9}$. It should be noted that museum specimens stored in solvents such as ethanol are not optimal for the extraction of skin lipids, and only fresh or freshly frozen carcasses should be considered as possible sources for extraction. Skin lipids are inert, which makes them stable on the surface of the skin and easy to extract ${ }^{7}$. In their signaling roles in reptile ecology, skin lipid cues are often deposited in harsh environments, but because of their robust chemical properties, such cues can retain their information value over long periods of time ${ }^{10,11,12}$. The extraction process solubilizes the lipids, using a nonpolar solvent (e.g., hexane, benzene, toluene) over an hours-long soak, followed by the evaporation of the solvent, to leave a measurable mass of lipid extract ${ }^{7,8}$. Skin lipids are highly miscible in nonpolar solvents, and a wide range of hydrocarbons can be extracted from a similarly diverse array of sources.

Fractionation is more laborious than extraction but serves to separate the total lipid extract into specific fractions via column chromatography, to aid in the purification and possible identification of the compounds therein ${ }^{1,6,7,8}$. The total lipid extract is bound to a substrate-based column, and then, individual eluates ("fractions") of solvent at specific volumes are passed sequentially through the column to elute sets of compounds 
from the lipid mixture that have a common polarity ${ }^{6,7,8}$. In lipid chromatography, the fractions progress in polarity at some standardized sequence by increasing the relative amount of polar solvent (e.g., diethyl ether) in nonpolar solvent (typically expressed as a percentage: $0 \%, 2 \%, 4 \%$ ether, etc. $)^{6,7,8}$. Though methods like thin-layer chromatography (TLC) can be used to separate lipids in a mixture and are simpler, column chromatography is preferred because it uses a closed system, is easy to control, can separate more concentrated mixtures, and is compatible with multiplexing for efficiency. In this manuscript, we describe several methods for extracting skin lipids of reptiles and, then, provide a standard protocol for isolating different sets of compounds based on polarity, using traditional column chromatography. In many research projects involving the isolation of chemical cues, the ultimate goal is to effect change in the receivers exposed to those cues. Whole lipid extracts or specific fractions can, then, be used in bioassays to determine any biological activity elicited by the compounds therein ${ }^{1,2,6,7}$. In basic biological research, for example, bioassays using specific fractions can reveal to researchers that a purified source of pheromones has been isolated, and then, methods for the identification of the target compounds can be pursued. From a wildlife management perspective, identification may not be the goal, and instead, the active fraction could be used in the field to attract conspecifics to traps or inhibit mate tracking in the nonnative habitat ${ }^{13,14}$.

\section{Protocol}

All procedures involving the use of vertebrates were approved by the Institutional Animal Care and Use Committee of James Madison University.

\section{Extraction}

\section{Shed skin extraction}

1. Gather approximately $30 \mathrm{~cm}^{2}$ of shed from a single reptile, removing the head and cloacal sections that can contaminate the samples Wear chloroprene gloves and clean the shed of debris.

2. Tare the balance with a bag or weigh boat and weigh the shed $( \pm 0.01 \mathrm{~g})$.

NOTE: The mass precision is determined by the precision of the balance. Shed skin mass is the standardization factor for extracted skin lipid mass (see below) and significantly covaries with extracted lipid mass.

3. Separate the shed into smaller $\left(5 \mathrm{~cm}^{2}\right)$ pieces and add them to a sealable glass container with a hexane-compatible lid (e.g., a glass mason jar with a metal lid or a lab flask with a PTFE lid). Decant enough hexane into the container to fully submerge the shed skin pieces. Seal the container.

CAUTION: Hexane is flammable, a respiratory irritant, and is associated with several short- and long-term health hazards. Procedures involving hexane are performed in a fume hood (laboratory) or outdoors (field) while wearing appropriate personal protective equipment (PPE) (e.g., splash goggles, chloroprene gloves, long sleeves, close-toed shoes).

4. Label the container(s) with a pencil or solvent-resistant pen. Leave the container(s) in the fume hood at room temperature overnight or up to $24 \mathrm{~h}$

5. Remove the shed pieces using clean metal forceps or tongs. Shake the pieces to retain any remaining hexane in the container. Allow the skin pieces to dry on paper towels; then, discard them. If multiple extractions are being performed, clean the tongs/forceps between each sample. To clean the tongs/forceps, rinse them in $\sim 50 \mathrm{~mL}$ of hexane in a beaker.

6. If the extract is not to be used immediately, decant or pipette the extract into a glass vial of appropriate volume, seal it with a PTFElined cap, label it, and store it at $-20^{\circ} \mathrm{C}$.

CAUTION: Because the extract contains hexane, store the vials in an explosion-proof freezer

\section{Dead whole animal extraction}

1. Record the snout-vent length (SVL; in centimeters) and mass (in grams) of the animal. If the total lipid or pheromone production per unit of skin surface area is to be determined, use a tailor's tape measure to obtain the maximum body circumference (in centimeters).

2. For the extraction, choose a container that has a diameter that is $1 / 3$ of the length of the animal. Position the carcass securely at the bottom of the container with the head and cloaca on top. Decant sufficient hexane into the container to maximize the submerged surface area of the body.

NOTE: If the head or cloaca become submerged for any amount of time in extraction, take note.

3. Secure the lid, label the container, and soak it in a fume hood at room temperature overnight or up to $24 \mathrm{~h}$.

4. When removing the carcass, use clean metal forceps or tongs. Retain the residual hexane on the carcass in the container by allowing it to drip from the body, not from the head or tail. Seal the container.

5. If the extract is not to be used immediately, decant the extract or pipette it into a glass vial of appropriate volume, seal it with a PTFElined cap, label it, and store it at $-20^{\circ} \mathrm{C}$.

\section{Lipid Mass Determination}

NOTE: The extracted lipid mass can be determined in one of two ways: with a glass vial or with a round-bottom flask, using a rotary evaporator.

1. Determine the extracted lipid mass via the glass vial method.

1. Use a preweighed vial of sufficient size $(7 \mathrm{~mL}, 22 \mathrm{~mL}, 50 \mathrm{~mL}$, etc.). Include the cap and the label in the total mass, or always weigh the vial without cap and label (markings on the label also add mass). Place the label on the neck of the flask to avoid water contact. NOTE: Evaporation in glass vials is efficient if the researcher has access to a gas manifold system where multiple vials can be evaporated simultaneously under an $\mathrm{N}_{2}$ stream.

2. Transfer the extract to the vial, using a glass pipette with a rubber bulb or, for large-volume extracts, an electronic pipette controller with a disposable $10 \mathrm{~mL}$ glass pipette. Rinse the container with $\sim 3 \mathrm{~mL}$ of hexane and transfer to the vial as well.

3. Evaporate the sample under a gentle $\mathrm{N}_{2}$ stream. Tilt the vial at an angle to enable a maximum solvent surface area. Condensate will form on the outside of the vial as the hexane evaporates.

NOTE: Rings of lipid will form in the vial as the hexane evaporates, so periodically swirl the extract.

4. Evaporate the sample to dryness; then, reweigh. Record the total lipid yield. 
NOTE: For analysis across different groups, standardize the lipid mass either to shed mass (lipid mass [in grams] divided by shed mass [in grams] $\times 100$ yields the percent lipid mass of the shed) or to the animal's SVL (lipid mass [in milligrams] divided by SVL [in centimeters]; yields the lipid mass per unit length). Larger animals produce more lipid, and many species are strongly sexually dimorphic, which imposes significant bias in the data.

2. Determine the extracted lipid mass via the rotary evaporator.

1. For a faster evaporation per individual sample, transfer the extract to a preweighed round-bottom flask and evaporate it using a rotary evaporator

1. If particulates are noticeable in the extract, filter the sample by placing a filter paper cone in the neck of the flask; then, transfer the extract to the flask and allow it to gravity filter. Dispose of the filter paper after the full extract is transferred. NOTE: Transfer the sample volume (up to $~ 80 \%$ flask volume) before rotary evaporation. Any bubbling of the extract into the condenser of the rotary evaporator causes contamination and requires the condenser to be cleaned. A bump trap can be placed between the flask and the neck of the condenser if bubbles or "bumping" occur in most samples.

2. Turn on the power to the rotary evaporator and water bath $\left(50^{\circ} \mathrm{C}\right.$; lower than the solvent boiling point). Turn on the cold-water flow to the condenser.

NOTE: The condenser of the rotary evaporator is connected to either a circulating chiller or a cold-water spigot venting to the drain. The flow rate can be slow if the water is cooler than ambient, to condense the solvent vapor leaving the flask and entering the condenser.

3. Turn on the vacuum source (water vacuum or pump). Ensure the vacuum pressure is sufficient to hold the flask to the neck of the condenser. Open the vent at the end of the condenser before connecting the flask. Slide the flask onto the neck of the condenser, close the vent to seal, and ensure the flask cannot disconnect from the condenser when the flask is released.

4. Lower the flask until $\sim 50 \%$ is submerged in the bath. Start the rotation of the flask at medium speed. If the vacuum, speed, condenser flow, and bath temperature are optimal, the solvent vapor leaving the flask will condense on the coil and drip into the recovery flask.

1. If the sample boils (e.g., large bubbles, rapid gassing), immediately reduce the vacuum and/or speed of the rotary evaporator. If the boiling continues, turn off the flask rotation, turn off the vacuum, raise the flask from the bath, and release the vacuum seal. Repeat steps 2.2 .3 and 2.2.4.

NOTE: If no solvent is collecting in the recovery flask but the extract is obviously evaporating, the vacuum is most likely nonoptimal and must be readjusted.

5. Evaporate the sample under the vacuum until $\sim 2 \mathrm{~mL}$ of solvent remains in the flask (large-volume extracts), or, if a preweighed flask is being used, evaporate until a bead of liquid with $a<1 \mathrm{~cm}$ diameter is visible in the bottom of the flask. Turn off the rotation and vacuum; then, raise the flask out of the bath.

6. Hold the flask neck as the vacuum seal is released; then, twist the neck to slide it from the condenser. If a large volume flask is being used, swirl the condensed extract in the flask to dissolve visible lipids; then, transfer the solution via pipette to a smaller-volume, preweighed flask. Add $3 \mathrm{~mL}$ of hexane to rinse the large flask, swirl it, pipette it to the smaller flask, and evaporate again (steps 2.2 .3 2.2.5).

7. The bead of liquid in the extract will solidify as the hexane evaporates. Once dry, allow it to reach room temperature. Lipids will form a translucent, white to yellow wax in the flask ( $\sim 5 \mathrm{~min})$. Weigh the flask to obtain the final mass.

NOTE: Depending on the nature of the lipids extracted, they will have either solid (e.g., wax) or semisolid (e.g., crude oil) properties, especially when fractionated lipids are evaporated (see below).

3. Solubilize the lipids in the recorded volume of hexane to yield $\geq 1 \mathrm{mg}$ of lipid per $1 \mathrm{~mL}$ of hexane. The working volume for progressing to chromatography is $\sim 5 \mathrm{~mL}$. If transferring from flask to vial, retain $2 \mathrm{~mL}$ of the total volume to rinse the flask after transferring the majority of the extract to the vial.

4. Label the vials, seal them with PTFE-lined caps, and store them at $-20^{\circ} \mathrm{C}$.

\section{Column Chromatography}

NOTE: To separate unknown compounds based on polarity into specific fractions, extracted lipid mass can be added to prepared liquid chromatography columns and fractionated using standard elution.

\section{Preparation of the column}

1. Depending on the lipid mass in the extract, use either a large- or a small-volume glass chromatography column with a Teflon stopcock. The column is either fitted with or fused to a fixed-volume reservoir ( $500 \mathrm{~mL}$ for a large column; $250 \mathrm{~mL}$ for a small column). Henceforth, this protocol only refers to a large-volume chromatography column fused to a reservoir. Thoroughly clean any new glassware and parts to be used in chromatography as described in section 4; then, rinse them with hexane or another nonpolar solvent

2. Fold a piece of fiberglass wool $(\sim 14 \mathrm{~cm}$ in length [L] $\times 4 \mathrm{~cm}$ in width [W]) repeatedly until a square of $\sim 4 \mathrm{~cm} \times 4 \mathrm{~cm}$ is formed. Use a wooden dowel rod longer than the column to position the fiberglass at the bottom of the column.

3. Secure the column in a hood to a standard ring stand with two clamps (e.g., swivel or modular), one above the stopcock and one beneath the neck of the reservoir. Level the column. Position the column at a height to allow sufficient working distance for the $500 \mathrm{~mL}$ beaker to fit easily under the column. Open the stopcock.

4. Pour washed and dried sand into the column, until a $\sim 3 \mathrm{~cm}$ of sand rests above the fiberglass. Place black or dark paper under the column and tap it gently. If sand falls from the column with every tap, the fiberglass barrier is insufficient. Repeat steps 3.1.2 - 3.1.4. NOTE: Use an unfolded paperclip taped to the end of a dowel rod to fetch the fiberglass from the bottom.

5. Place the $500 \mathrm{~mL}$ beaker under the column. Slowly decant $\sim 25 \mathrm{~mL}$ of hexane from a $100 \mathrm{~mL}$ beaker or graduated cylinder into the column reservoir to wet the sand. Close the stopcock when there is $\sim 0.5 \mathrm{~cm}$ of hexane above the sand.

6. Weigh out neutral alumina. For each small column, use $\sim 50 \mathrm{~g} ; \sim 175 \mathrm{~g}$ for each large column. Pour the alumina into a $1 \mathrm{~L}$ Erlenmeyer flask. Limit $400 \mathrm{~g}$ of alumina per $1 \mathrm{~L}$ flask. 
7. To activate the alumina (activity III), pipette deionized water of a volume equal to $6 \%$ of the alumina mass (i.e., for $100 \mathrm{~g}$ of alumina, add $6 \mathrm{~mL}$ of water). Add the water as drops throughout the alumina.

8. Cover the flask with aluminum foil or a cork. Vigorously swirl (do not shake) to evenly disperse the charge. Continue until no visible clumps remain.

NOTE: The flask will warm as the water reacts with the alumina, which is to be expected.

9. Add hexane until the alumina is completely covered, with $\sim 0.5 \mathrm{~cm}$ of hexane above the alumina. Swirl the flask to form a slurry.

10. Place a vented funnel in the reservoir of the column and a $500 \mathrm{~mL}$ glass beaker beneath the column. Open the stopcock. Swirl the alumina and steadily pour it into the column. Ensure the alumina is settling evenly in the column and no large bubbles or cracks are forming in the alumina column. Gently tap the side of the column to evenly settle the alumina.

NOTE: Additional hexane must be added for additional pours, to maintain the slurry. The hexane collecting in the beaker under the column can be reused if the glass beaker was clean at the start.

11. The column is formed when the top of the alumina is stable and $\sim 4 \mathrm{~cm}$ beneath the neck of the column at the base of the reservoir. Use a pipette to rinse the inside of the reservoir with hexane for residual alumina. Using a funnel, gently add a second layer of sand on top of the alumina, to $\sim 1 \mathrm{~cm}$ beneath the reservoir.

NOTE: Hexane will flow from the column as the stopcock remains open. Do not let the column dry out. If the column dries out, the process must begin again, starting at step 3.1.2. The protocol can be stopped here if the columns are prepared a day before fractionation. Firmly place a cork in the top of the reservoir or cover it tightly with foil. Fill the reservoir with $\sim 100 \mathrm{~mL}$ of hexane. Tighten the stopcock.

\section{Fractionation of the lipid extract}

NOTE: Regardless of column size, fractions of lipid extract can be collected individually and pooled based on their fraction polarity or discarded completely if they do not contain any known/identified target compounds. Rounds of replicate elution ( $n=3$ volumes) are passed through the column to ensure the sufficient elution of the lipids. Follow Table 1 for large-column elution (with an extract mass $>30$ mg) or small-column elution (with an extract mass $<30 \mathrm{mg}$ ). A tailored elution protocol for isolating only methyl ketones is in Table 2.

1. Either remove the remaining hexane at the top of the column, using a large-volume pipette, or allow the hexane to drip out into a clean beaker. Leave $\sim 3 \mathrm{~mL}$ of hexane above the sand at the top of the column.

NOTE: The collected hexane is pure if it only contacted clean glassware and can be recycled in the first fraction.

2. Transfer the lipid extract to the column, using a long glass pipette. Slowly pipette the extract to avoid disturbing the sand layer. Rinse the extract vial or flask with $\sim 5 \mathrm{~mL}$ of hexane and transfer it to the column. NOTE: If the extract was stored at $-20^{\circ} \mathrm{C}$, the lipids will be precipitated. Warm the vial until no more precipitate is visible.

3. Open the stopcock and allow the sample to load into the column. If the extract has a large mass (>30 mg), a yellowish band may be visible in the alumina, just below the sand at the top. Collect the flow through in a waste beaker as this hexane is no longer clean. Close the stopcock when $\sim 3 \mathrm{~mL}$ remains on top of the sand.

CAUTION: The lipid in the extract, now bound to the alumina and column, cannot dry out or the sample will be lost.

4. Place a preweighed and labeled round-bottom flask $(100 \mathrm{~mL}, 250 \mathrm{~mL}$, or $500 \mathrm{~mL})$ beneath the column before pouring the first fraction. Fractions to be discarded can be collected in a common glass container.

5. Prepare the first fraction ( $100 \%$ hexane:0\% diethyl ether [heretofore, "ether"]) in a graduated cylinder. If preparing fractions in advance, cover with foil or stopper with cork. NOTE: Fractions progress in polarity; therefore, the cylinder does not need to be rinsed between fractions.

6. Add the first fraction to the reservoir by pouring via a vented glass funnel directed to a side of the reservoir to avoid disturbing the sand layer. Open the stopcock to start the elution. Close the stopcock when $\sim 3 \mathrm{~mL}$ of hexane remains above the sand at the top of the column.

NOTE: Never stop an elution within an individual fraction by closing the stopcock before the majority of the eluate has been collected. Between fractions, do not leave the stopcock closed for longer than $\sim 1 \mathrm{~h}$ because delays can alter the quality and repeatability of the elution.

7. Repeat steps 3.2.4 - 3.2.6 for fractions 2 and 3. Collect the fractions in appropriately sized flasks that allow headspace for rotary evaporation, especially if the fractions are being pooled.

8. Prepare a fourth fraction ( $2 \%$ ether) and add it to the reservoir. Place the next flask under the column, open the stopcock, and collect the fourth fraction. Prepare a fifth fraction; then, continue as needed. NOTE: It is possible to evaporate the first fraction(s) via rotary evaporation while collecting successive fractions.

9. For preparing fractions to be used in GC-MS analyses, obtain a fraction mass using a 0.01 or $0.1 \mathrm{mg}$ precision balance. Solubilize the lipid fractions to yield $1 \mathrm{mg}$ of lipid per $1 \mathrm{~mL}$ of hexane.

\section{Cleaning}

1. Leave the stopcock open and invert the column in the waste beaker. The alumina and sand will run out of the column; alternatively, shake to expedite the process. Use a dowel rod to fetch the fiberglass wool if it is stuck (avoid scratching glass) or blow it out with an $\mathrm{N}_{2}$ stream.

2. Dissemble and clean all glassware, using laboratory-grade detergent in hot water in a plastic tub with a glassware brush (with hair or plastic bristles). Wash $3 x$. Rinse well with plenty of warm water until the glass is no longer slick to the touch.

3. Rinse the glassware and tools $3 x$ with deionized water. Place them on a rack to air-dry. To accelerate the drying, add a small volume of acetone $(3 \mathrm{~mL})$ to the glassware, swirl, and allow it to air-dry or run it under an $\mathrm{N}_{2}$ stream.

\section{Representative Results}

Following extraction, the total lipid mass is the first type of data that can be acquired through the protocol presented here. However, total lipid mass values should never be analyzed without some attempt to standardize the obtained values. Several approaches can be used, but we recommend either standardizing the extracted lipid mass to the animal's SVL (in centimeters) or to the mass of the shed skin that was extracted. The former results in a lipid-mass-per-length value and the latter will be a proportion of source mass. The reason for the standardization is that 
larger animals naturally produce more skin lipids because they have a greater total skin surface area. Figure 1A exemplifies this association, where the extracted skin lipid mass scales linearly with the mass of shed skin extracted. Once standardized to the total shed skin mass, this linear relationship is completely removed (Figure 1B).

Following fractionation, the same standardization approach can be used with the masses of the individual fractions (Figure 2). In this sample data set, each fraction is not contributing equally to the total extracted lipid mass: neutral lipids (fractions 1 - 3 ) are the dominant set of compounds by mass proportion compared to each set of more polar lipids (fractions $4-6,7$ - 9, and $10-12$ ).


Figure 1: Relationships between extracted lipid mass and input material. (A) In reptiles, the relationship between the total shed skin mass and the extracted skin lipid mass is positively correlated $(P<0.001)$. (B) When the extracted skin lipid mass is standardized to the total shed mass (the lipid mass divided by the shed mass), this relationship is no longer present $(P=0.46)$. Please click here to view a larger version of this figure.



Figure 2: Standardized fraction masses following elution. Using column chromatography, skin lipid extracts can be eluted into fractions, based on compound polarity. The fraction mass is, then, expressed as a proportion of the total lipid extract (fraction mass [in milligrams] divided by total lipid extract mass [in milligrams) to determine differences between fractions or experimental groups ${ }^{8}$. The bars represent the means. The top error is SEM; the bottom error is $95 \% \mathrm{Cl}$. Individual data points are provided for clarity. Please click here to view a larger version of this figure. 


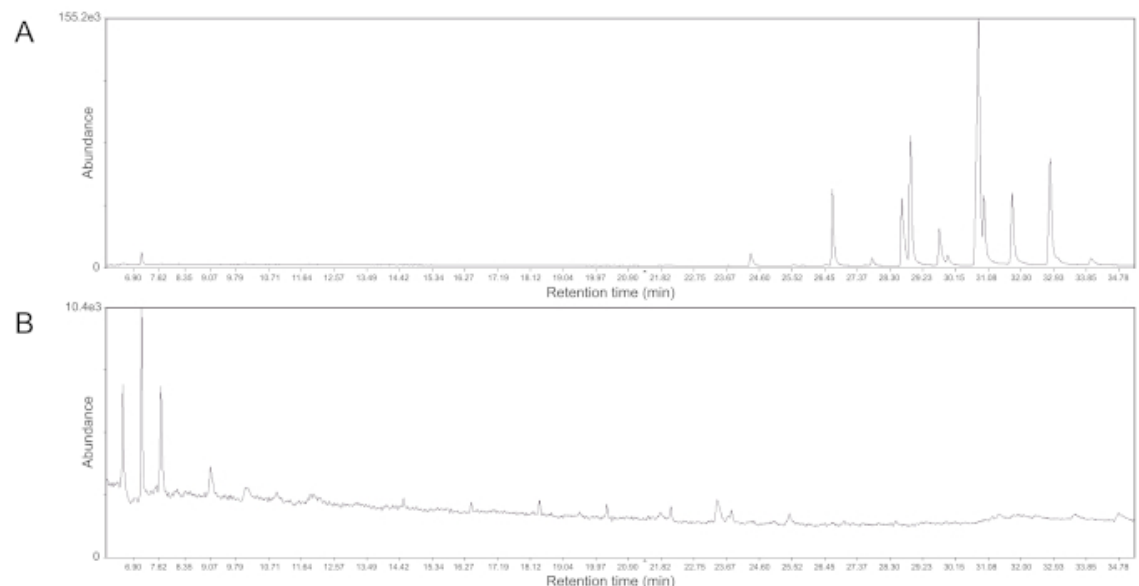

Figure 3: Representative gas chromatographs of methyl ketone fractions. (A) With the proper elution, methyl ketones are the most abundant compounds in fraction 7 from Table 2 and can be seen as couplets of peaks (retention time $=24-34$ min). (B) Fraction 6 from Table 2, however, only yields nontarget compounds. This same result can occur when the polar solvent used in the mobile phase is expired or if the column is stopped for long periods of time $(>1 \mathrm{~h})$ between fractions. Note the difference in molecular abundance between the two traces. Please click here to view a larger version of this figure.

\begin{tabular}{|l|l|l|}
\hline Fraction & Hexane $(\mathrm{mL})$ & Diethyl ether $(\mathrm{mL})$ \\
\hline $1,2,3$ & $100[30]$ & $0[0]$ \\
\hline $4,5,6$ & $98[29.4]$ & $2[0.6]$ \\
\hline $7,8,9$ & $96[28.8]$ & $4[1.2]$ \\
\hline $10,11,12$ & $92[27.6]$ & $8[2.4]$ \\
\hline $13,14,15$ & $84[25.2]$ & $16[4.8]$ \\
\hline
\end{tabular}

Table 1: Standard elution volumes for skin lipid fractionation. Solvent volumes and percentages are given for both large-volume (e.g., 250 $\mathrm{mL}$ ) and [small-volume] (e.g., $100 \mathrm{~mL}$ ) chromatography columns using alumina (activity III). Hexane is the carrier solvent; diethyl ether is the mobile phase.

\begin{tabular}{|c|c|c|c|}
\hline Fraction & Hexane ( $m L)$ & Diethyl ether $(m L)$ & Notes \\
\hline $1,2,3$ & 30 & 0 & elute; do not collect \\
\hline 4,5 & 28.8 & 1.2 & elute; do not collect \\
\hline $6,7,8$ & 28.8 & 1.2 & $\begin{array}{l}\text { collect individually; majority of } \\
\text { methyl ketone mass will be in } \\
\text { fraction } 7 \text {; GC-MS quality control } \\
\text { checks should be run on } 6 \text { and } \\
8 \text { to ensure proper elution of the } \\
\text { ketones }\end{array}$ \\
\hline
\end{tabular}

Table 2: Modified elution scheme for garter snake methyl ketones. These volumes are for use with a small-volume chromatography column and with the brand of alumina currently available. The eluted fraction positive for methyl ketones has a strong bioactivity in field assays with wild, courting male garter snakes ${ }^{1,7}$. To confirm the presence of methyl ketones in the target fractions, samples can be diluted to $1 \mathrm{mg} / \mathrm{mL}$ and analyzed via GC-MS. Figure 3 provides representative chromatograms for both positive and negative results following elution.

\section{Discussion}

The extraction of skin lipids in reptiles can be applied to living or dead skin, in addition to shed skin, which offers versatility in the experimental application of this technique. Further, extractions of skin lipids can be done in the field, to enable a dynamic application of the method to a wide range of biologists ${ }^{2,13}$. Extraction of skin lipids is simple; therefore, it is easy to scale up extractions as needed per experiment or design, and practitioners need not have significant expertise to execute the methods. The only limiting factors for scaling up are the availability of fume hood space, an abundance of clean, sealable glassware, and solvent storage space.

Skin lipid extract fractionation can be tailored to a researcher's needs and, therefore, has similar flexibility to lipid extraction. For example, neutral lipids can be eluted and then discarded, to result in target fractions that may purify compounds of interest or simplify bioassays. Fractionation can be performed at multiple scales within and across lipid samples. For example, multiple columns can be run simultaneously to make the process more efficient. Or, only a portion of a total lipid extract can be fractionated on a small column to, thus, spare reagents and time. Fractionation is primarily limited by the mass of the total lipid extract and the precision of the equipment available to the researcher. For example, if the researcher has a balance with a $10.0 \mathrm{mg}$ precision, the determination of the fraction mass and, therefore, the accuracy of the sample preparation 
for the GC-MS analysis is significantly, if not completely, impeded. The same is true for the glassware. If the researcher has a large-volume column for fractionation but has a small total lipid mass to separate, the elution or separation of the compounds will progress but will require a significant wastage of solvents, reagents, time, and potentially, the target compounds themselves.

It is advised to perform a quality control check before determining the elution scheme, as seen in Table 2, and decide what fractions may be discarded. To confirm the elution of the desired lipids, a column can be run where each fraction, 1 - 15, is collected individually and then analyzed using GC-MS. In Figure 3, representative gas chromatograph traces show that methyl ketones from garter snakes only elute from the column in a specific fraction. By performing this quality control step, a modified elution scheme can be developed for a given species to ensure the maximum yield of the compounds of interest. Changes in the materials, such as the supplier or lot of the alumina or the age of the diethyl ether, will absolutely result in differences in elution that should be controlled for by performing a quality control test.

The techniques described are limited chiefly by the chemical nature of the cues that can be obtained. Primarily, these methods only allow researchers to isolate and separate long-chain lipids from the skin of reptiles. Many species of reptiles use airborne and/or proteinaceous cues as chemical signals, and the described methods are incompatible with isolating said cues. Further, nonpolar solvents will not extract aqueous cues from the surfaces of reptiles or sources of cue deposition (e.g., cage water, fecal matter, aquatic substrate) that may indeed contain abundant chemical signals. Appropriate methods for capturing these types of cues are available to researchers (e.g., solid-phase microextraction [SPME] for volatile cues and high-performance liquid chromatography [HPLC] for aqueous cues), although, like the methods described above, there is a technical learning curve.

Most importantly, the utility of the final chemical mixture to the researcher should guide the methods used. For example, if a researcher wants to know if a male focal animal can distinguish between the cues produced by male vs. female conspecifics in a targeted bioassay, extraction is the only method needed ${ }^{2,3}$. If the identification of sexually dimorphic compounds is the goal, however, the extract ought to be purified, to enable greater confidence in assigning identifications to specific molecules or groups of molecules via chemical analysis ${ }^{1,6,9,11}$. However, to even conduct chromatography with a lipid source, a significant mass of starting extract is required so that measurable fraction masses can be obtained; otherwise, the pooling of samples can be pursued but is not optimal ${ }^{14}$.

Future developments of this protocol include measures to utilize and adapt the procedure for more reptilian species. Additional noninvasive methods of extracting skin lipids are also being developed.

\section{Disclosures}

The authors have nothing to disclose.

\section{Acknowledgments}

The development of these methods, especially shed skin lipid extraction, occurred during cooperative agreements (14-7412-1061-CA, 15-7412-1155-CA, and 16-7412-1269-CA) between James Madison University (JMU) and the U.S. Department of Agriculture's Animal and Plant Health Inspection Service (APHIS). M.R.P. acknowledges the contributions of the following students to the development of the shed skin methods: S. Patel (Washington and Lee University [WLU]), J. Zachry (WLU), R. Flores (JMU), J. Noll (JMU), and S. Ashton (JMU).

\section{References}

1. Mason, R.T. et al. Sex pheromones in snakes. Science. 245 (4915), 290-292 (1989).

2. Mason, R.T., Parker, M.R. Social behavior and pheromonal communication in reptiles. Journal of Comparative Physiology A. 196 (10), 729-749 (2010).

3. Martín, J., López, P. Pheromones and chemical communication in lizards. In Reproductive Biology and Phylogeny of Lizards and Tuatara. Edited by Rheubert, J.L., Siegel, D.S., Trauth, S.E., 43-77, CRC Press. Enfield, NJ (2014).

4. Mayerl, C., Baeckens, S., Van Damme, R. Evolution and role of the follicular epidermal gland system in non-ophidian squamates. AmphibiaReptilia. 36 (3), 185-206 (2015).

5. Mathies, T., Levine, B., Engeman, R., Savidge, J.A. Pheromonal control of the invasive brown treesnake: potency of female sexual attractiveness pheromone varies with ovarian state. International Journal of Pest Management. 59 (2), 141-149 (2013).

6. Parker, M.R., Patel, S.M., Zachry, J.E., Kimball, B.A. Feminization of male brown treesnake methyl ketone expression via steroid hormone manipulation. Journal of Chemical Ecology. 44 (2), 189-197 (2018).

7. Parker, M.R., Mason, R.T. Pheromones in snakes: history, patterns, and future research directions. In Reproductive Biology and Phylogeny of Snakes. Edited by Sever, D., Aldridge, R., 551-572, CRC Press. Enfield, NJ (2011).

8. Parker, M.R., Mason, R.T. Low temperature dormancy affects the quantity and quality of the female sexual attractiveness pheromone in redsided garter snakes. Journal of Chemical Ecology. 35 (10), 1234-1241 (2009).

9. Parker, M.R., Mason, R.T. How to make a sexy snake: estrogen activation of female sex pheromone in male red-sided garter snakes. Journal of Experimental Biology. 215 (5), 723-730 (2012).

10. Alberts, A.C. Constraints on the design of chemical communication systems in terrestrial vertebrates. American Naturalist. 139, S62-S89 (1992).

11. Martín, J., Ortega, J., López, P. Interpopulational variations in sexual chemical signals of Iberian wall lizards may allow maximizing signal efficiency under different climatic conditions. PLoS ONE. 10 (6), e0131492 (2015).

12. Baeckens, S. et al. Environmental conditions shape the chemical signal design of lizards. Functional Ecology. 32 (2), $566-580$ (2018).

13. Greene, M.J., Mason, R.T. Chemically mediated sexual behavior of the brown tree snake, Boiga irregularis. Ecoscience. 5 (3), $405-409$ (1998). 
14. Mason, R.T. Integrated pest management: The case for pheromonal control of habu and brown tree snakes. In Snakes and Man: Controlling Pest Species for Conservation and Human Health. Edited by Rodda, G., Chiszar, D., Sawai, Y., Tanaka, H., 196-205, Cornell University Press. Ithaca, NY (1998).

15. Pruett, J.A. et al. Evolutionary interactions between visual and chemical signals: chemosignals compensate for the loss of a visual signal in male Sceloporus lizards. Journal of Chemical Ecology. 42 (11), 1164-1174 (2016). 